\title{
The Malleable Mesenchyme- Elastofibroma
}

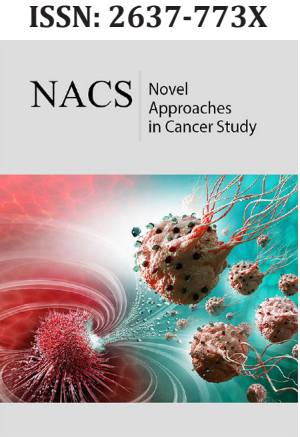

*Corresponding author: Anubha Bajaji, Histopathologist in A.B. Diagnostics, New Delhi, India

Submission: 傮 : September 16, 2020

Published: 眥 November 20, 2020

Volume 5 - Issue 4

How to cite this article: Anubha Bajaji. The Malleable MesenchymeElastofibroma. Nov Appro in Can Study. 5(4). NACS.000617. 2020. DOI: $10.31031 /$ NACS.2020.05.000617

Copyright@ Anubha Bajaji, This article is distributed under the terms of the Creative Commons Attribution 4.0 International License, which permits unrestricted use and redistribution provided that the original author and source are credited.

\author{
Anubha Bajaj* \\ Histopathologist in A.B. Diagnostics, India
}

\section{Introduction}

Elastofibroma dorsi is an uncommon, benign, subcutaneous, poorly circumscribed, nodular, preponderantly subscapular soft tissue neoplasm representing as a pseudo-tumour or a tumour-like lesion. The neoplasm is essentially composed of collagen and coarse, enlarged elastic fibres. Additionally, nomenclated as elastofibroma dorsi, the tumefaction can be contemplated as a reactive, mesenchymal hyperplasia with aberrant elastogenesis. Elastofibroma characteristically demonstrates elastic fibres intermixed with collagenous stroma and mature adipose tissue. Jarvi et al. [1] initially described the neoplasm in 1959 at the $12^{\text {th }}$ congress of Scandinavian Pathologists. The condition was subsequently published in 1961 [1]. With anomalous imaging manifestations or doubtful tumour discernment, close monitoring, pertinent imaging or evaluation of cogent tissue specimen is recommended.

\section{Disease Characteristics}

Typically, elastofibroma is localized within subcutaneous tissue and skeletal muscle of the dorsal surface of the shoulder joint, abutting the inferior border of scapula. On account of the characteristic location, the neoplasm is cogitated as elastofibroma dorsi. Elastofibroma dorsi arises from connective tissue of the chest wall and is configured of collagen bundles [2]. Classic site of emergence of elastofibroma is scapular region between sixth and eighth ribs and apex or inferior angle of scapula beneath the serratus anterior, latissimus dorsi and levator scapulae muscles. Majority (90\%) of neoplasms are predominantly situated within periscapular, subscapular or infra-scapular region beneath rhomboid major and latissimus dorsi muscle, deep-seated to the serratus anterior. Additionally, sites such as the axilla, oral cavity, ischial tuberosity, greater trochanter, posterior elbow, stomach, rectum, omentum, orbit, hand, foot, deltoid muscle, infra-olecranon, thoracic wall, spinal canal, gluteal and inguinal region, thigh, periosteum, mediastinum or cornea are incriminated $[2,3]$. The neoplasm is commonly discerned within the fifth decade, in adults exceeding $>50$ years with an age range of 51 years to 79 years and a mean age of tumour discernment at 68.4 years. Tumefaction discerned in children and infants is extremely exceptional [2,3]. A female predilection is observed. Elastofibroma commonly appears within subscapular region of thoracic fascia in around $24 \%$ of adult females and roughly $11 \%$ of adult males exceeding 58 years. History of intense physical labour or repetitive activities can be elicited. Elastofibroma probably represents an exaggerated response to trauma or friction (Figure 1). Racial predilection is denominated with enhanced disease incidence in Japanese individuals [2,3]. Although bilateral, the lesion is frequent discerned upon the right side. Bilateral lesions are discerned in roughly $33 \%$ of the subjects although neoplasms may be non- synchronous. Besides concomitant infra-scapular and infra- olecranon lesions, multiple lesions of elastofibroma are uncommonly delineated $[2,3]$. Although considered exceptional, elastofibroma demonstrates a variable disease prevalence of around $2.73 \%$, as discerned with computerized tomography and nearly $24 \%$ upon autopsy of the geriatric population. Tumour monitoring for a duration of one month to 30 months with a mean period of 8.6 months demonstrates an absence of residual clinical symptoms or localized tumour reoccurrence $[2,3]$. As the enlarged, soft tissue pseudo-tumour is deep-seated within deep fascia, often exceeds $>5$ centimeters magnitude and is intensely adherent to circumscribing bone or soft tissue, possible occurrence of soft tissue sarcoma requires exclusion $[2,3]$. 


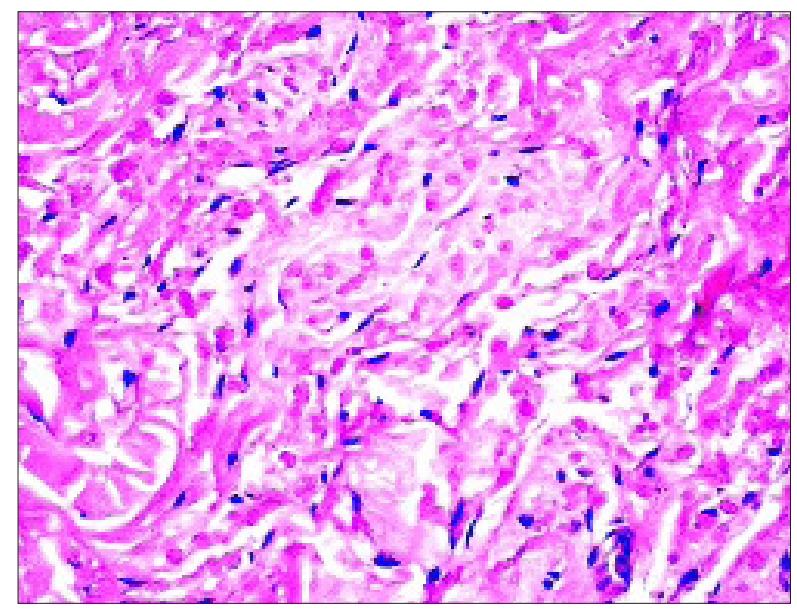

Figure 1: Elastofibroma delineating fascicles of elastic tissue, skeletal muscle fibres, collagen tissue and mature adipose tissue cells.

Disease Pathogenesis

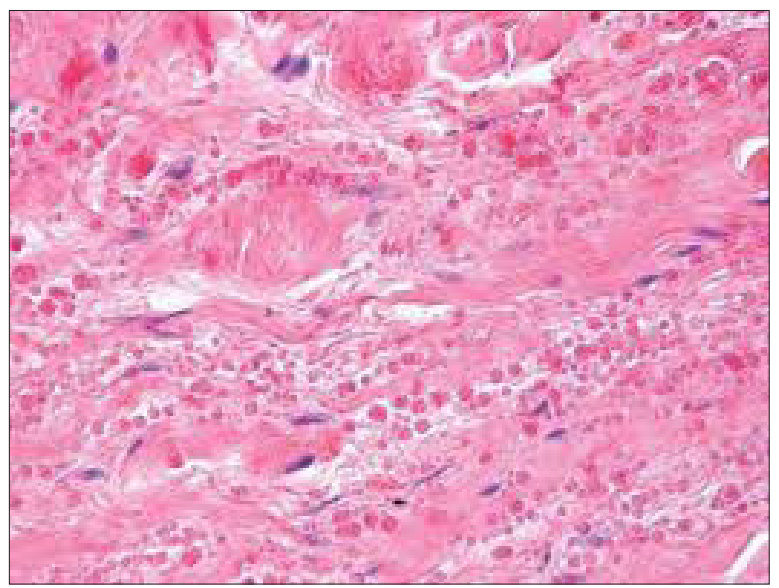

Figure 2: Elastofibroma depicting bands of elastic fibres, mature adipose tissue, collagenous tissue and mature skeletal muscle.

Diverse environmental and genetic theories are posited for engenderment of elastofibroma. Although disease pathogenesis is obscure, it is considered to be multifactorial. Elastofibroma is not a true neoplastic process and may be engendered by repetitive micro-trauma or friction of the inferior pole of the scapula with the thoracic wall. Repetitive, mechanical trauma induces reactive hyper-proliferation and degeneration of fibro-elastic tissue $[3,4]$. Elastofibroma arises in regions of limited mechanical friction and can appear within dominant and non-dominant sides. Elderly individuals commonly demonstrate an elastofibroma, the frequency of which is enhanced amongst manual workers and the right side [4]. Micro-trauma or mechanical theory is not comprehensively applicable and inadequately hypothesizes incurrence of elastofibroma (Figure 2). Vascular insufficiency may engender degenerative alterations and elastofibroma originates as a gradual neoplastic process [4]. Monoclonal proliferation with genomic instability is also incriminated in the emergence of elastofibroma. Alternatively, hereditary enzyme defects may induce reactive hyper-proliferation of fibro-elastic tissue [2,4]. Genetic predisposition with familial neoplasms can possibly denominate certain instances as a cogent family history is documented in nearly $33 \%$ of elastofibromas $[2,4]$. Thus, it can be summarized that elastofibroma demonstrates a multifactorial aetiology and a singular theory of disease emergence is insufficient.

\section{Clinical Elucidation}

Commonly, a firm, palpable, non-tender, subscapular mass of extended duration and limited mobility is discerned. Clinical symptoms appear within 3 months to 60 months with a mean duration of 20 months. Bilateral lesions can occur [4]. Elastofibroma represents as an asymptomatic, painless, or painful, infiltrative, gradually progressive, subcutaneous tumefaction arising in the region of inferior angle or tip of scapula. Incriminated, elderly individuals represent a swelling, discomfort, occasional pain, and functional restriction of daily or strenuous physical activities [4]. Symptoms can be engendered by impingement of brachial plexus. Cogent clinical symptoms manifest as swelling, discomfort, snapping of scapula and occasional pain $[4,5]$. Tumour tissue can extend into deep-seated subcutaneous tissue, in between skeletal muscles and adhere to the periosteum of middle ribs. Upon examination, a firm, deep-seated, fixed, non-tender lump, adherent to the abutting rib cage, is denominated within specific sites such as infra-scapular region. Associated lymph node enlargement is absent $[4,5]$. Histological evaluation may be deferred in asymptomatic individuals with a typical clinical representation and definitive radiological discernment [4].

\section{Histological Elucidation}

On gross examination, a firm, rubbery, ill defined, adherent, nonencapsulated neoplasm composed of fibrous tissue admixed with yellowish streaks of elastin is discerned. Tumour magnitude varies from 3 centimetres to 15 centimeters with an average dimension of 7 centimeters. Cut surface is grey/white and well circumscribed (Figure 3). Elastofibroma is intermixed with macroscopic zones of fibrous tissue and mature adipose tissue. Infiltration of adjacent skeletal muscle bestows a variegated, reddish, whitish or yellowish discoloration to the tumefaction [5,6]. On cytology, smears are minimally cellular and display diagnostic globules of eosinophilic, elastic tissue disseminated within a collagenous matrix. Altered, elastic fibres demonstrate a green- yellow auto fluorescence when exposed to ultraviolet light [5]. On histology, a hypo-cellular tumour composed of benign fibroblasts admixed with eosinophilic collagen and elastin fibres is exemplified. Microscopically, numerous characteristics, dense, randomly distributed, branched or unbranched, elastic fibres are disseminated within a collagenous stroma. The fibres can be focally fragmented into miniature, bead-like, spheroids or eosinophilic globules. Elastic fibres are intermingled with few, bland, fibroblasts, myofibroblasts and mature adipocytes. Also, mature adipose tissue cells are interspersed between benign fibroblasts [5,6]. Collagen bundles are enunciated which alternate with enlarged, thickened, eosinophilic, 
elastic cylinders delineating a dense, centric core. Elastic fibres may be fragmented into linear globules, thus configuring the classic "beads on a string" appearance. Irregular inter-digitations into circumscribing adipose tissue are denominated. Heterogeneous, dense, eosinophilic elastic fibres intermingled with mature adipose tissue, skeletal muscle and collagenous fragments can be observed $[5,6]$. The neoplasm is composed of slightly diverse amino acids as compared to elastin. Collagen type I to collagen type III normally articulate the neoplastic tissue as type II collagen in usually restricted to articular cartilage and ocular structures $[5,6]$. Thus, abundant fibrous collagenous tissue, occasional fibroblasts and innumerable eosinophilic elastic fibres are commingled with islets of mature adipose tissue. A tumour free surgical perimeter is desirable (Figure 4). On ultrastructural examination, elastofibroma demonstrates irregular, spherical masses of electron dense, preelastin encompassing minimally dense, elastin which is engendered within rough endoplasmic reticulum of adjacent fibroblasts. Cell cylinders are composed of immature, amorphous, elastic tissue with a centric core and mature fibres [5]. On cytogenetic analysis, anomalies of chromosome $1 \mathrm{p}$ and Xq1 are exemplified. The neoplasm demonstrates an Xq12-q22 or \#19 chromosomal gain in an estimated $30 \%$ of tumefaction $[5,6]$.

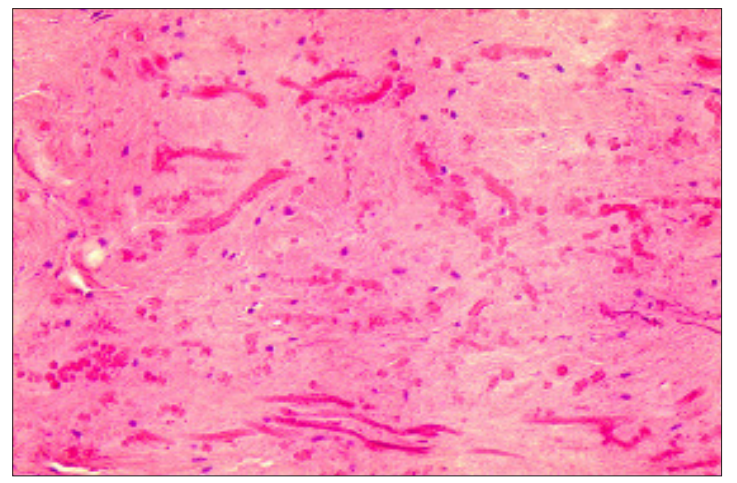

Figure 3: Elastofibroma demonstrating bands of elastic tissue, fibroblasts, myofibroblasts and collagen fibres with skeletal muscle.

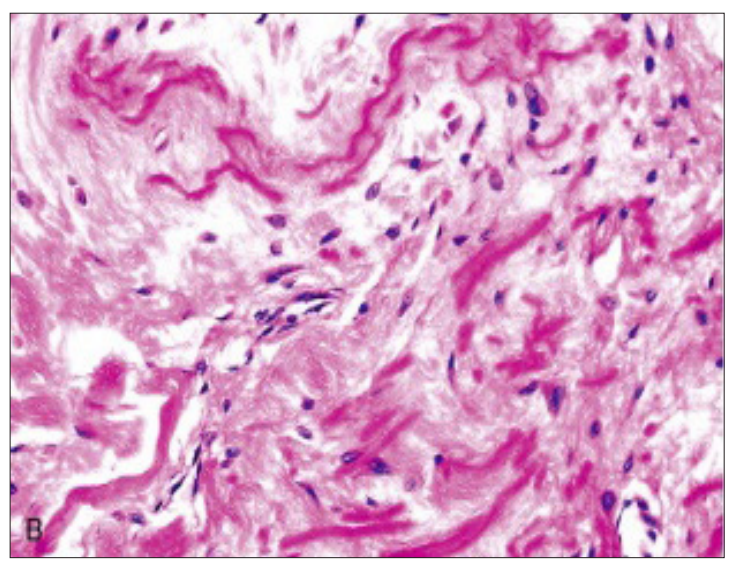

Figure 4: Elastofibroma exhibiting fascicles of elastic tissue, fibroblasts, myofibroblasts, mature adipose tissue cells and collagen fibrils.

\section{Immune Histochemical Elucidation}

Elastofibroma is immune reactive to vimentin. Spindle-shaped cellular component is immune reactive to CD34. Elastic stains exemplify elastic fibres with a dense core and irregular margins (Figure 5). The tumour is immune non-reactive to S100 protein, desmin, smooth muscle actin (SMA) and p53 [2,3].

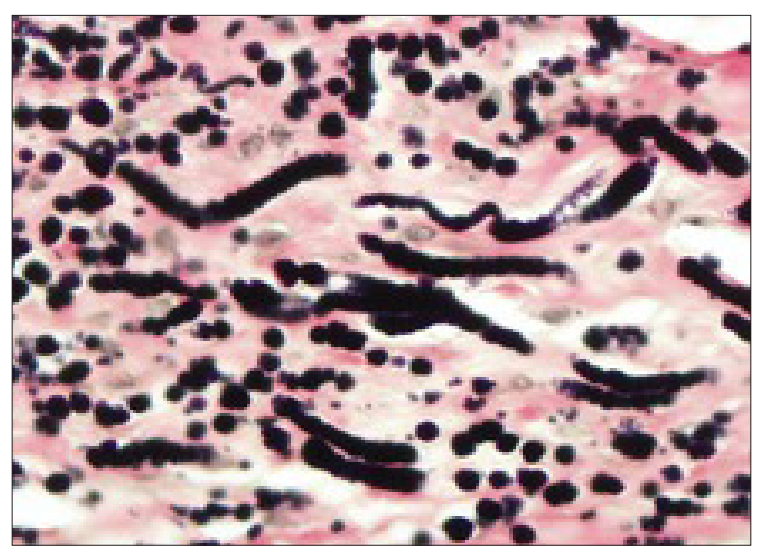

Figure 5: Elastofibroma delineating branched and unbranched elastin fibrils intensely stained dark brown to black with elastin stain.

\section{Differential Diagnosis}

Elastofibroma requires a segregation from neoplasia such as primary or secondary sarcoma, neurofibroma, desmoid tumour, fibro-lipoma, nuchal fibroma or malignant fibrous histiocytoma [6].

a) Desmoid fibromatosis demonstrates enhanced cellularity, infiltrates adjoining skeletal muscle and is devoid of elastic fibres.

b) Fibro-lipoma is a neoplasm depicting an absence of elastic fibres.

c) Nuchal fibroma occurs in younger individuals beneath $<55$ years [6]. Lesions appear between scapula and vertebrae. The neoplasm is composed of dense fascicles of collagen although elastic fibres are absent [7].

d) Malignant fibrous histiocytoma exhibits nuclear and cellular atypia and pleomorphism, storiform tumour configuration, multinucleated giant cells and an absence of elastic fibres [7].

\section{Investigative Assay}

Elastic fibres can be highlighted with histochemical stains such as Weigert's elastic stain. Masson trichrome demonstrates an intense cellular staining. On elastin stains, branched and unbranched elastic fibres with a centric, dense core and serrated periphery are intensely enunciated. Immune reactivity to antielastin stains highlights elastic fibres, which fluoresce with ultraviolet illumination [8]. Elastic fibres thus denominated can be segregated from amyloid deposits, which exhibits an applegreen birefringence with Congo red staining. Elastic tissue can be eliminated with elastase digestion [7,8]. Typical lesions arising in typical locations can be adequately categorized with imaging techniques. Thus, pertinent tissue sampling may not be warranted 
in specific instances [8]. Plain radiographs can be unremarkable or demonstrate an elevated scapula wherein the scapulo- thoracic space may appear enlarged. An opacity between dual scapula may be recognized in the absence of bony lesions or associated calcifications. Adjoining soft tissue can be dense. Preoperative tumour discernment can be managed with presumptive assessment upon computerized tomography (CT) or magnetic resonance imaging (MRI) in concurrence with cogent clinical findings. Ultrasonography, computerized tomography (CT) and magnetic resonance imaging (MRI) can be suitably employed for probable discernment of elastofibroma (Figure 6). Radiological assessment with computerized tomography (CT) or magnetic resonance imaging (MRI) can categorically delineate bilateral lesions $[7,8]$. Ultrasonography depicts alternating patterns of hypoechoic and hyperechoic linearity which may be parallel to the chest wall. Colour Doppler demonstrates an insignificant vascular outflow [8]. Computerized tomography (CT) of the thorax depicts typical characteristics of an un-encapsulated, heterogeneous, lenticular neoplasm with hypodense fragments which appear iso-dense to adjacent muscular articulations and soft tissue masses situated underneath latissimus dorsi. Contrast enhanced computerized tomography (CT) demonstrates solid, well defined, unilateral or bilateral tumefaction predominantly within the infra-scapular region, buried deep beneath the serratus anterior muscle. Tumour mass is iso-dense, in contrast to encompassing skeletal muscle and exhibits a multi-layered appearance with hypodense, intrinsic or invasive fatty streaks. Infiltration of abutting anatomic structures or associated bony lesions is absent [8]. Positron emission computerized tomography (PET CT) frequently enunciates mild to moderate uptake of fluoro-deoxy glucose, contingent to enhanced tumour vascularity, fibroblastic proliferation and inflammation within the tumefaction [7]. Upon magnetic resonance imaging (MRI), unilateral neoplasm appears as a poorly circumscribed, heterogeneous, soft tissue mass. On account of occasional enhancement with gadolinium contrast medium, exclusion of a soft tissue sarcoma is required [7,8]. On magnetic resonance imaging (MRI), a poorly circumscribed, un-encapsulated, lenticular soft tissue mass with signal intensity similar to skeletal muscle along with regions of alternating enhanced and minimal signal intensity upon T1 weighted and T2 weighted imaging is denominated. Bilateral lesions enunciated within typical locations can depict aforesaid signal intensities upon T1 weighted imaging and T2 weighted spin echo sequences, a feature which is diagnostic of elastofibroma $[7,8]$. Magnetic resonance imaging (MRI) with T1 weighted and T2 weighted axial and coronal imaging scans display a signal intensity akin to adjoining skeletal muscle (Figure 7). Focal, linear hyperintensity is exemplified within the tumefaction following signal intensity of adipose tissue with an absence of localized tumour infiltration [7,8]. A moderately heterogeneous mass with hyperintense signal upon T2 weighted imaging is demonstrated. Fusion of diffusion- weighted sequence and T2 weighted imaging delineate an intense restriction of the tumefaction. Diffusion-weighted magnetic resonance imaging (DWI) displays enhanced tumour cellularity and benign characteristics of the neoplasm $[7,8]$.

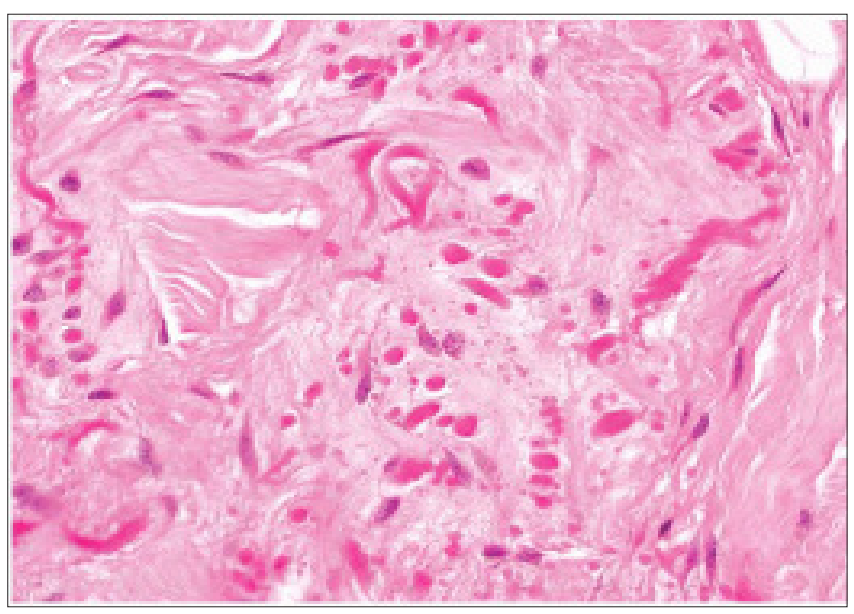

Figure 6: Elastofibroma exemplifying elastin fibres, collagen fibres, fibroblasts, myofibroblasts, minimal chronic inflammation and few adipose tissue cells.

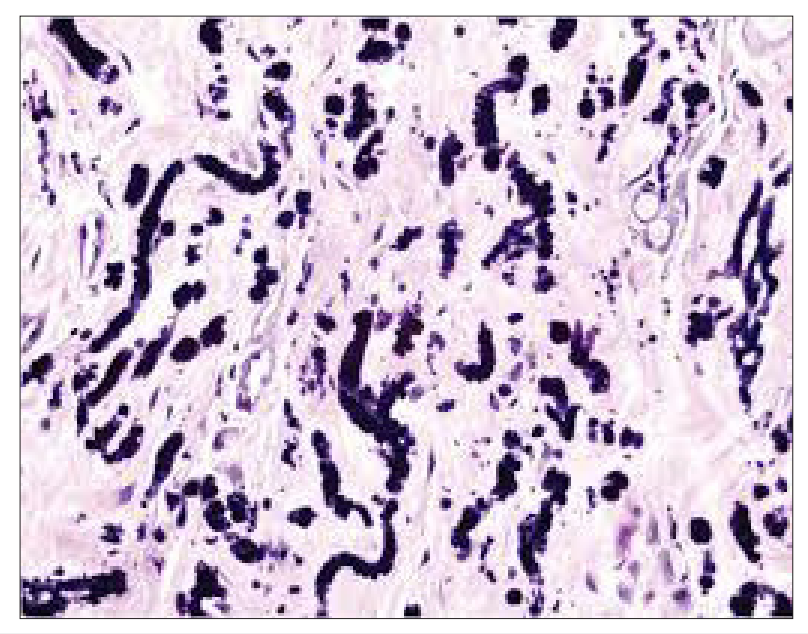

Figure 7: Elastofibroma depicting branched and unbranched elastic fibres stained intense, dark brown to black with elastin stain.

\section{Therapeutic Options}

Symptomatic neoplasm with mild clinical representation can be managed with surgical excision of neoplasm. Comprehensive surgical eradication of the neoplasm is recommended in symptomatic subjects or in individuals with cosmetic concerns. Radical resection or excision with a broad, tumour-free perimeter is circumvented as marginal resection is generally sufficient, curative and a preferred treatment strategy $[7,8]$. Surgical extirpation is imperative in managing the benign tumefaction arising in an elderly population. Peri-scapular region is extremely vascular and incidence of postoperative haematoma requires critical consideration, evaluation and treatment (Figure 8). With the appearance of unilateral lesions, bilateral neoplasms require exclusion and dual tumefaction is preferably extirpated within a singular sitting. Surgical eradication of the neoplasm is curative [7,8]. Non-surgical treatment methods can also be adopted. Asymptomatic, incidental elastofibromas may not mandate a surgical intervention in the 
majority of instances [8]. Tumour is usually devoid of reoccurrence with a median follow up of 32 months and range of monitoring from 12 months to 80 months. Associated complications are absent. Localized tumour reoccurrence is uncommon and arises due to incomplete surgical excision as the tumour's perimeter is often indistinct. As malignant metamorphoses is undocumented, thus regular monitoring is unrequired $[7,8]$.

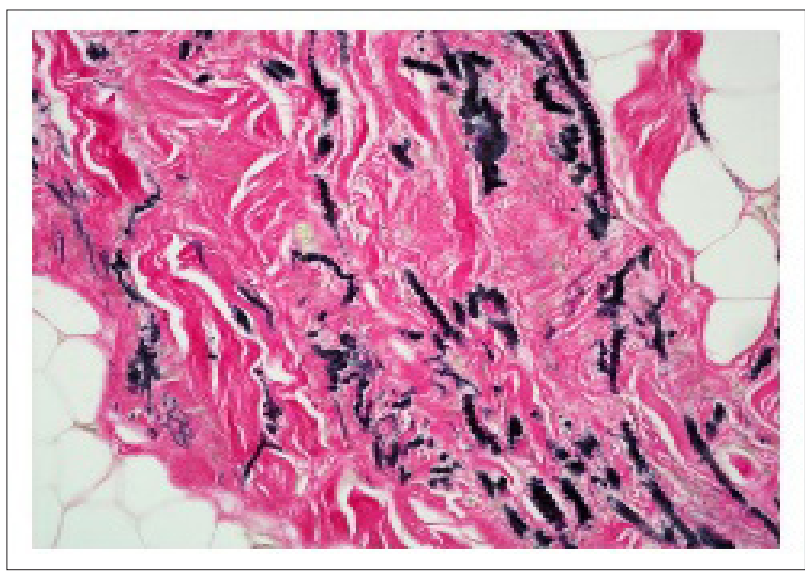

Figure 8: Elastofibroma stained with Weigert's elastin stain exhibiting branched and unbranched elastic fibres.

\section{References}

1. Jarvi OH, Saxen AE (1961) Elastofibroma dorse. Acta Pathol Miicrobiol Scand 51(Suppl 144): 83-84.

2. Temel U, Akgul AG, Salih T (2020) Diffusion MR - a new diagnostic tools for elastofibroma dorsi. Sisli Etfal Hastan Tip Bul 54(1): 103-107.

3. Al Dandan O, Hassan A, Mona M, Jumanah M, Haidar A, et al. (2020) Concomitant bilateral elastofibroma in the infrascapular and gluteal regions- a report of a rare case. BMC Musculoskeletal Disord 21(1): 16.

4. Tepe M, Polat MA, Calisir C, Inan U, Murat B (2019) Prevalence of elastofibroma dorsi on CT - is it really an uncommon entity? Acta Orthop Traumatol Turc 53(3): 195-198.

5. Bulam H, Sezgin B, Kemal B, Nesibe C (2015) A one-year old boy with paraspinal elastofibroma- the youngest diagnosed elastofibroma. Ann Thorac Surg 100(1): 302-304.

6. Deveci MA, Ozbarlas HS, Kıvılcım E, Omer S, Mustafa T, et al. (2017) Elastofibroma dorsi- clinical evaluation of 61 cases and review of literature. Acta Orthop Traumatol Turc 51(1): 7-11.

7. Olchowy C, de Delas'-Vigo MA, Manolo P, Nikaoly C, Rosa D (2008) Triple elastofibromas located in the supra-and infra scapular regions - a case report. Skeletal Radiol 47(4): 569-573.

8. Cevolani L, Casadei R, Vanel D, Gambarotti M, Donati D (2017) Elastofibroma of the gluteal region with a concomitant contralateral lesion- case report and review of literature. Skeletal Radiol 46(3): 393397. 